\title{
Variabilidad espacial y temporal del suministro larval de mitílidos en el Seno de Reloncaví, sur de Chile
}

\author{
Spatial and temporal variability of mytilid larval supply in the Seno de Reloncaví, southern Chile
}

\author{
Aura Barria ${ }^{1}$, Paulina Gebauer ${ }^{1}$ y Carlos Molinet $^{2}$
}

\begin{abstract}
${ }^{1}$ Centro i Mar, Universidad de Los Lagos, Casilla 557, Puerto Montt, Chile. pgebauer@ulagos.cl ${ }^{2}$ Programa de Investigación Pesquera, Instituto de Acuicultura, Universidad Austral de Chile, sede Puerto Montt, Casilla 1327, Puerto Montt, Chile

Abstract.- The larval supply in benthic species is an important component for successful recruitment, and knowledge of its processes helps in understanding population dynamics. The present work describes temporal and spatial variations in larval supply of Mytilus chilensis and Choromytilus chorus in 3 sites on the coast of Seno de Reloncaví, and their relation with environmental variables (temperature, salinity and wind component). The presence of competent larvae of $M$. chilensis and $C h$. chorus presented variations in space and time, explained by the variables year, month and location, and additionally for $M$. chilensis, the east-west wind component. The magnitude of larvae availability for both species was explained too by the year, month and locality variables, suggesting this variable, effects on a local scale. The greatest abundance of competent larvae, observed on the north-east coast of Seno de Reloncaví, was associated with low salinity and temperature values, suggesting the intrusion of waters from the Reloncaví Estuary. Extensive banks of mytilids have been identified in this zone, which may be acting as a larva source for the Seno de Reloncaví.
\end{abstract}

Key words: Competent larvae, abiotic variables, Mytilus chilensis, Choromytilus chorus

\begin{abstract}
Resumen.- El suministro larval en especies bentónicas es un componente importante que contribuye al éxito del reclutamiento y por lo tanto su conocimiento permite entender la dinámica de las poblaciones. En el presente trabajo se estudió la variación temporal y espacial del suministro larval de Mytilus chilensis y Choromytilus chorus en 3 localidades en la costa del Seno de Reloncaví y su relación con variables ambientales (temperatura, salinidad y componente del viento). La presencia de larvas competentes de $M$. chilensis y Ch. chorus presentó variabilidad temporal y espacial, la que fue explicada por las variables año, mes y localidad, y adicionalmente para $M$. chilensis por el componente de viento esteoeste. La magnitud de la disponibilidad de larvas para ambas especies, fue explicada también por las variables año, mes y localidad, sugiriendo esta variable, efectos de escala local. Una mayor abundancia de larvas competentes observada en la costa noreste del Seno de Reloncaví fue asociada a bajos valores de salinidad y temperatura, lo que sugiere la intrusión de agua del estuario del Reloncaví. En esta zona se han identificado extensos bancos de mitílidos que podrían estar actuando como fuente de larvas para el Seno de Reloncaví.
\end{abstract}

Palabras clave: Larvas competentes, variables abióticas, Mytilus chilensis, Choromytilus chorus

\section{INTRODUCCIÓN}

En invertebrados bentónicos con ciclo de vida complejo el reclutamiento es el resultado de varios eventos que ocurren en distintas escalas temporales y espaciales: el suministro larval; el asentamiento y metamorfosis; y la supervivencia de los juveniles recién asentados. Es por ello que no siempre es clara la relación entre el suministro larval y la magnitud del reclutamiento (Pineda et al. 2010).

El suministro larval corresponde a la densidad de larvas competentes en las cercanías del hábitat apropiado para asentamiento (Cameron \& Schroter 1980, Harrold et al. 1991). Este es un importante proceso pre-asentamiento que contribuye como agente estructurador de los ensambles y comunidades de invertebrados bentónicos marinos, e incluso puede ser más importante que los procesos post- asentamiento tales como competencia, depredación y disturbios físicos (Todd 1998).

Por otra parte, la relación entre el tamaño del stock desovante y el reclutamiento es el resultado de diversos procesos, desacoplados tanto en sus escalas espaciales y temporales. Así por ejemplo, el desove, supervivencia larval y llegada de larvas competentes depende principalmente de procesos de gran escala, mientras que la receptividad de los sitios para el asentamiento, en general depende de las condiciones locales (pequeña escala) (Todd 1998, Pineda 2000, Orensanz et al. 2006). 
El suministro larval restringe el asentamiento y reclutamiento de los organismos (Rilov et al. 2008, Pineda et al. 2010) y es afectado por la supervivencia larval, el comportamiento larval y los procesos físicos lo cual tiene consecuencias para la conectividad poblacional. Por esto, el estudio del suministro de larvas es uno de los elementos clave para entender el resultado del reclutamiento y la conectividad de las poblaciones (Pineda et al. 2010).

Considerables variaciones espaciales a distintas escalas $(\mathrm{cm}$ a $\mathrm{km})$ en el asentamiento y reclutamiento en varios estudios dentro de una misma región biogeográfica han sido atribuidas al suministro larval (Connell 1985, Petraitis 1991, Bertness et al. 1992, Hunt \& Schiebling 1996). Específicamente, Porri et al. (2006a) al examinar la disponibilidad larval y asentamiento del molusco bivalvo Perna perna concluyeron que los parches de asentamiento observados en escalas de cientos de metros es debido a que las larvas son transportadas preferentemente a algunas localidades más que a otras, probablemente por efecto de la morfología y topografía local.

En el mar interior del sur de Chile Choromytililus chorus (Molina, 1782) y Mytilus chilensis (Hupé, 1854) son mitílidos frecuentes que forman densos 'bancos' sobre fondos duros y fangosos hasta una profundidad de 5-7 m, aunque existen registros hasta $25 \mathrm{~m}$ de profundidad (Lorenzen et al. 1979, Zagal \& Hermosilla 2001). Mytilus chilensis es el mitílido de mayor importancia comercial en Chile ( $\approx$ se capturan 220.000 tons, SERNAPESCA 2010). El desove de ambas especies ocurre principalmente en primavera verano (Chaparro \& Winter 1983) y el desarrollo larval se extiende por 19 a 45 días para M. chilensis (Toro \& Sastre 1995, Toro et al. 2004, Ruiz et al. 2008), siendo de 27 días a $19^{\circ} \mathrm{C}$ para Ch. chorus (Bellolio et al. 1996) bajo condiciones de laboratorio. A pesar de la importancia de ambas especies y al desarrollo de la acuicultura de $M$. chilensis sustentada en la captación natural de semillas, existe escasa información publicada respecto al suministro larval de estos mitilidos y a los factores relacionados a éste, en zonas naturales de asentamiento y en centros de cultivo.

Por otro lado, la zona donde se desarrolla la mitilicultura, es un complejo sistema de fiordos y canales

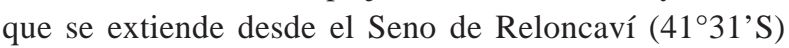
hasta Magallanes (56²0’S) (Pickard 1971, Silva et al. 1995, Silva et al. 1998). Diversos autores sugieren que en este tipo de ambiente se generan gradientes de densidad que pueden actuar como barreras físicas, que pueden influir en la posición de las larvas en la columna de agua y en su transporte final a las zonas de asentamiento (Mann et al. 1991, Shanks 1995, Young 1995, Ardisson \& Bourget 1997, Dobretsov \& Miron 2001).

El objetivo del presente trabajo fue estudiar la variabilidad espacio-temporal del suministro de larvas competentes de $M$. chilensis y Ch. chorus y su relación con variables ambientales, en el intermareal rocoso de la costa noreste del Seno de Reloncaví.

\section{Materiales y MÉTODOS}

\section{ÁreA de EsTudio}

El estudio fue realizado en el Seno de Reloncaví, ubicado en el extremo norte del mar interior de la Patagonia Noroccidental que forma parte de un complejo sistema estuarino con una columna de agua estratificada (Pickard 1971, Silva et al. 1997, 1998; Dávila et al. 2002, ValleLevinson et al. 2007). La temperatura superficial (0-6 m) varia entre $9^{\circ} \mathrm{C}$ y $18^{\circ} \mathrm{C}$ en invierno y verano, respectivamente, y la salinidad entre 20 y 32 (Iriarte et al. 2007). Bajo $7 \mathrm{~m}$ de profundidad la temperatura varia entre 11 y $12^{\circ} \mathrm{C}$ y la salinidad es $\approx 33$. La productividad primaria presenta estacionalidad, con mayores valores de biomasa en primavera (septiembre) y otoño (marzo-abril) (Iriarte et al. 2007).

Los muestreos fueron realizados en la costa noreste del Seno de Reloncaví, Puerto Montt, Chile, en el intermareal de 3 localidades: Pelluhuín (4129'57's,

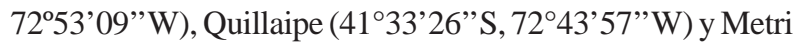

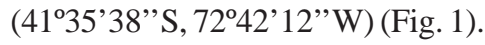

\section{RECOLECCIÓN DE MUESTRAS}

La disponibilidad de larvas competentes (LC) de mitílidos en las cercanías de las áreas de asentamiento fue muestreada desde septiembre a marzo durante 2 temporadas (años) (T2008: 2007-2008 y T2009: 2008-2009). En la zona intermareal media (2,5 m sobre la línea de marea baja) de cada localidad se fijaron 10 trampas (Tr) de larvas construidas de $35 \mathrm{~cm}$ de una manga plástica tejida con un monofilamento de 0,2 $\mathrm{mm}$ de diámetro; estas mangas plásticas fueron enrolladas formando almohadillas de aproximadamente $8 \mathrm{~cm}$ de diámetro, las que correspondieron a las trampas utilizadas en el estudio. Las 10 trampas fueron retiradas y reemplazadas cada 3 días. En el laboratorio se identificaron y contaron las LC de $M$. chilensis y Ch. chorus retenidas en cada trampa, siguiendo la descripción de Ramorino \& Campos (1983). 


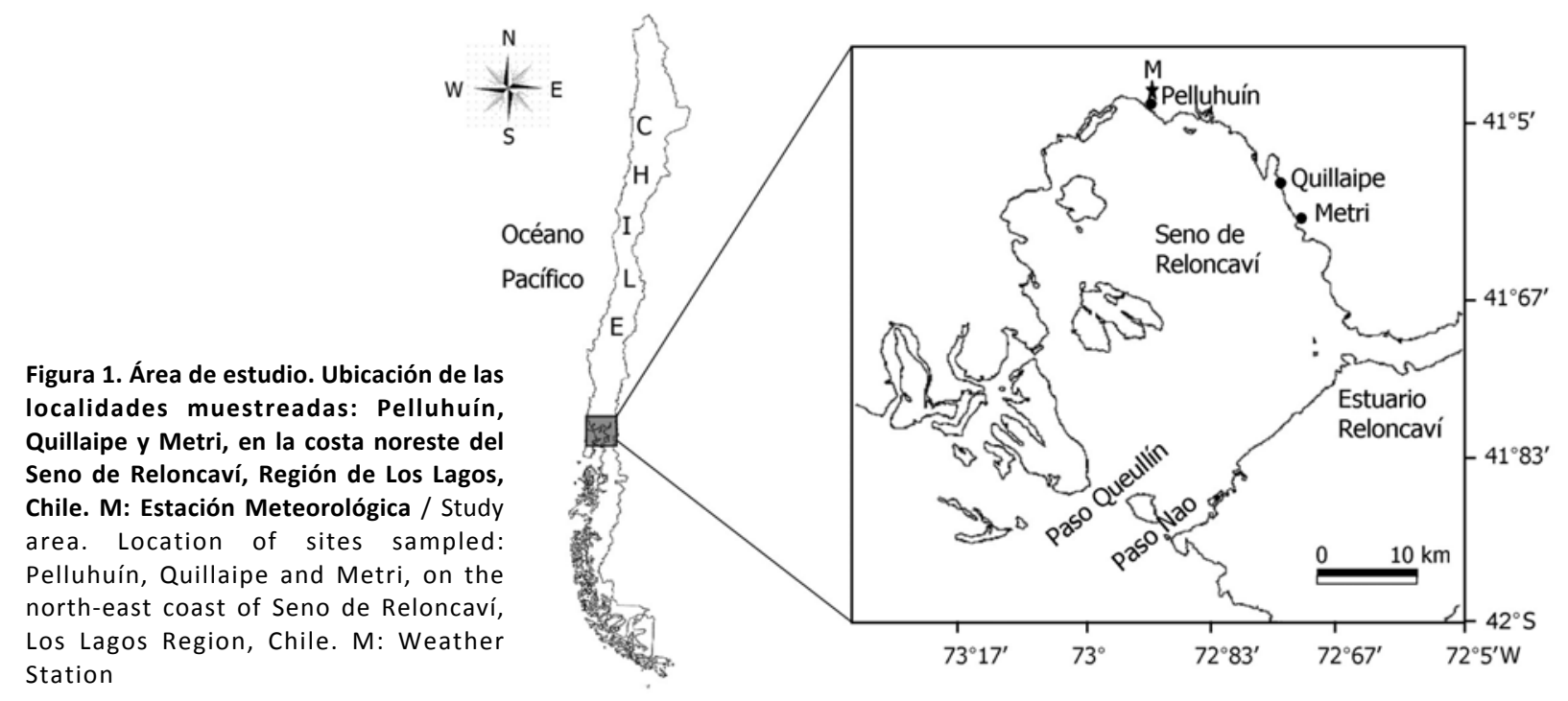

El criterio adoptado para definir a los individuos competentes, en el presente estudio, fue que presentaran desarrollo completo de la Prodisoconcha II y sin desarrollo de la Disoconcha, cuyo crecimiento indica que el individuo ya realizó la metamorfosis (Ramorino \& Campos 1983).

En cada localidad se instalaron equipos Star Odii, Iceland para registro continuo de temperatura y salinidad, los que fueron configurados para registrar estas variables cada 15 min. Los equipos fueron instalados a una distancia de $50 \mathrm{~m}$ de la costa a $2 \mathrm{~m}$ de profundidad. Lamentablemente, solo se obtuvo registros para todo el periodo de muestreo en Metri, ya que en las otras 2 localidades no fue posible recuperar los equipos o los datos. La velocidad y dirección del viento fue registrada por un anemómetro (Wind Monitor - AQ, Campbell Scientific ${ }^{\circledR}$ Inc.), ubicado en la zona noreste del Seno de Reloncaví (Fig. 1). La amplitud de marea se obtuvo a través de información entregada por el Servicio Hidrográfico y Oceanográfico de Chile para Puerto Montt. El componente de viento fue expresada como norte-sur y este-oeste considerando magnitud y dirección siguiendo a Emery \& Thompson (1997), entregando como resultado una variable continua cuyos valores pueden ser positivos (norte o este) o negativos (sur u oeste) dependiendo de la dirección predominante del componente. Las variables mencionadas fueron analizadas como el promedio de las mediciones durante los 3 días que las trampas estuvieron instaladas en el intermareal.

\section{ANÁLISIS DE DATOS}

Para modelar la abundancia de LC en las cercanías de los sitios de asentamiento para cada una de las especies estudiadas se utilizó una aproximación al modelo general lineal delta gamma (GLM) (sensu Stefánsson 1996) basado en el método de Aitchison-Pennington (Aitchison 1955, Pennington 1983), esto debido a la gran cantidad de valores 0 en las muestras, lo que impidió aplicar un análisis de varianza sin violar los supuestos de homogeneidad de varianza. Por otro lado, si hubieran sido excluidos los ceros del análisis, se estaría ignorando parte importante de la información. Las variables predictoras fueron año (T2008, T2009), mes (octubre, noviembre, diciembre, enero y febrero), localidad (Metri, Pelluhuín y Quillaipe), componente de viento norte-sur y componente de viento este-oeste. Un test a posteriori de Tukey para comparaciones múltiples fue aplicado simultáneamente a todos los factores del modelo para evaluar la significancia estadística entre cada nivel a través de los parámetros estimados siguiendo a Hothorn et al. (2008), usando la librería ‘multcomp' en R 2.14.0.

Un modelo específico fue ajustado para $M$. chilensis en la localidad de Metri ya que en ésta se obtuvieron registros de temperatura y salinidad durante el periodo de estudio. Para esto se utilizó el promedio de los registros de temperatura y salinidad obtenidos durante los 3 días que las trampas estuvieron instaladas en el intermareal. En esta aproximación estadística la densidad positiva observable $\left(d_{p}\right)$ es considerada una variable al azar con 
un máximo de masa de probabilidad en el origen. La media $\left(a_{p}\right)$ fue calculada ignorando las observaciones nulas, lo cual conduce a una estimación independiente sobre la probabilidad de la presencia de LC en una muestra $(\hat{p})$ para luego estimar una densidad corregida $\left(\hat{d}_{c}\right)$ por la siguiente relación:

$$
\hat{d}_{c}=\hat{p} \cdot \hat{d}_{p} \quad v\left(\hat{d}_{c}\right)=\hat{p}^{2} \cdot v\left(\hat{d}_{p}\right)+v(\hat{p}) \cdot \hat{d}_{p}{ }^{2}
$$

Las densidades medias positivas fueron calculadas a través del procedimiento de un modelo general mixto (Wolfinger \& O’Connell 1993) asumiendo una distribución gamma de los errores y usando una función 'log-link'. La distribución gamma de los errores fue seleccionada por tener mayor flexibilidad en grupo de datos no normales (McCullagh \& Nelder 1989).

Los datos de presencia/ausencia de LC de las muestras fueron analizadas como una variable dicotómica (0/1) y usada para estimar $\hat{p}(d>0)$ dentro del procedimiento de un modelo general mixto. Para esto, se asumió una distribución binomial de los errores y una función 'logitlink' (Kleinbaum et al. 1998).

Para elegir entre los modelos alternativos relacionados con las variables explicatorias (tales como la variable categórica mes de muestreo, o una variable continua 'salinidad') se utilizó el modelo que produjo el menor valor del criterio de información Akaike (AIC) (Burnham \& Anderson 2004). Todos los modelos generales lineales fueron construidos y analizados usando los paquetes 'stats' y 'car' en R.2.14.0 (Fox \& Sanford 2010, R Development Core Team 2011).

Aplicando la ecuación de estado a las variables temperatura, salinidad y profundidad se obtuvo la densidad, buscando una mejor caracterización de cada sitio muestreado y proveyendo parsimonia en el modelo. Sin embargo, de acuerdo al AIC, los modelos que incluyeron temperatura y salinidad tuvieron mejor ajuste que aquellos que solo incluyeron la densidad del agua, por lo que se utilizó ambas variables en el modelo.

La relación entre las series de tiempo de disponibilidad de LC para cada especie en todas las localidades (Metri, Quillaipe y Pelluhuín) para cada año de muestreo fue analizada mediante correlaciones cruzadas (Legendre \& Legendre 1998). Las series de tiempo de disponibilidad de LC de ambas especies fueron normalizadas $(\ln (x+1))$, la tendencia removida y los datos suavizados por medias móviles, 3 puntos (Chatfield 2004). Los desplazamientos $(\operatorname{lag}=\lambda)$ representan un periodo de 3 días ( $\lambda$ : $1=3$ días $)$ mientras que $\lambda$ : 0 indica la relación de la disponibilidad de LC para el mismo día de muestreo. En función del periodo en que se registró el $90 \%$ de las larvas competentes se acotaron las series de tiempo desde el 05 de noviembre del 2007 al 22 de enero del 2008 (T2008) y desde el 04 de noviembre del 2008 al 21 de enero del 2009 (T2009) para M. chilensis, y para Ch. chorus: desde el 05 de noviembre del 2007 al 09 de febrero del 2008 (T2008) y desde el 04 de noviembre del 2008 al 08 de febrero del 2009 (T2009).

\section{Resultados}

VARIABILIDAD TEMPORAL Y ESPACIAL DEL SUMINISTRO DE LARVAS COMPETENTES (LC)

En ambas temporadas (años) y en todas las localidades muestreadas, LC de Mytilus chilensis fueron más abundantes que LC de Choromytilus chorus, aunque durante T2008 se observó mayor abundancia de LC en ambas especies $\left(136,3 \pm 153,4\right.$ y 1,8 $\pm 2,5 \mathrm{LC} \mathrm{Tr}^{-1} 3 \mathrm{~d}^{-1}$, respectivamente) que durante T2009 $(41,5 \pm 61,7$ y $0,3 \pm$ $0,4 \mathrm{LC} \mathrm{Tr}^{-1} 3 \mathrm{~d}^{-1}$, respectivamente) (Tabla 1, 2). Las larvas competentes de $M$. chilensis y Ch. chorus estuvieron presentes con mayor frecuencia en la localidad de Metri, la localidad más cercana al estuario del Reloncaví, que en las otras localidades (comparación a posteriori de Tukey, $P<0,05)$ y no se detectaron diferencias en la presencia de larvas entre las localidades de Quillaipe y Pelluhuín $(P>$ 0,05) (Tabla 1, 2, Fig. 2-3 A-F).

La mayor abundancia de LC de Mytilus chilensis se observó entre noviembre y enero (90\%) en ambas temporadas (Fig. 2-3 A, C, E), con diferencias significativas en la presencia y densidad de larvas entre los meses. Para el caso de la densidad de larvas competentes las diferencias se detectaron en casi todos los meses (comparación a posteriori de Tukey, $P<0,05$ ) excepto entre octubre y enero, y octubre y febrero $(P>0,05)$. En T2008 se observaron 2 modas en las series de Pelluhuín y Metri, a mediados de noviembre y diciembre, (Fig. 2 A, E). En T2009 se observó sólo una moda, durante diciembre en todas las localidades (Fig. $3 \mathrm{~A}, \mathrm{C}, \mathrm{E}$ ). Al igual que para M. chilensis, la abundancia de LC de Choromytilus chorus en T2008 y T2009 se concentró desde el inicio de noviembre hasta enero en todas las localidades (Fig. 2-3 B, D, F). Durante la T2008 Ch. chorus presentó solo una moda durante noviembre y en la siguiente temporada una moda bien marcada en noviembre y una débil en enero, especialmente en Metri (Fig 2, 3 B, D, F). 
Tabla 1. Modelo seleccionado para explicar la variabilidad de larvas competentes por trampa de $M$. chilensis en el área de estudio, usando una aproximación al modelo general lineal Delta-gamma basado en el método de Aitchison-Pennington. La presencia/ausencia de larvas competentes fue analizada como una variable dicotómica (0/1) asumiendo distribución binomial de los errores y usando una función 'logit-link'. La densidad positiva de larvas competentes, fue calculada a través del uso de un modelo lineal general mixto, asumiendo una distribución gamma de los errores y usando una función log-link / Model selected to explain the variability of competent larvae per trap of $M$. chilensis in the study area, using a general linear model approach (GLM) to Delta-gamma based AitchisonPennington method. Presence/absence of competent larvae was analyzed as a dichotomous variable $(0 / 1)$ assuming binomial distribution of errors and using a logit-link function. Positive density of competent larvae was calculated using a mixed general linear model, assuming a gamma distribution of errors and a log-link function

\begin{tabular}{ccccc}
\hline $\begin{array}{c}\text { Grados de } \\
\text { libertad }\end{array}$ & $\begin{array}{c}\text { Desvianza } \\
\text { residual }\end{array}$ & $\begin{array}{c}\text { Grados de } \\
\text { libertad residual }\end{array}$ & Desvianza & $\operatorname{Pr}\left(>\chi^{2}\right)$ \\
\hline
\end{tabular}

Variable respuesta: Presencia/ausencia de larvas competentes de $M$. chilensis

$\begin{array}{llllll}\text { Modelo nulo } & & & 248 & 311,15 & \\ \text { Año } & 1 & 71,62 & 246 & 239,47 & 0,001 \\ \text { Mes } & 4 & 89,01 & 242 & 150,47 & 0,001 \\ \text { Localidad } & 2 & 26,20 & 240 & 124,22 & 0,001 \\ \text { Componente de viento } & 1 & 14,61 & 238 & 108,65 & 0,001\end{array}$

Este-Oeste

Variable respuesta: Densidad positiva de larvas competentes de M. chilensis

$\begin{array}{lrrrrr}\text { Modelo nulo } & & & 169 & 457,04 & \\ \text { Año } & 1 & 147,77 & 167 & 309,26 & 0,001 \\ \text { Mes } & 4 & 95,27 & 163 & 213,98 & 0,001 \\ \text { Localidad } & 2 & 11,43 & 161 & 202,55 & 0,017 \\ \text { Componente de viento } & 1 & 1,80 & 159 & 199,60 & 0,245\end{array}$

Tabla 2. Modelo seleccionado para explicar la variabilidad de larvas competentes por trampa de Ch. chorus en el área de estudio, (ver Tabla 1 para mayores explicaciones) / Model selected to explain the variability of competent larvae per trap of Ch. chorus in the study area, (for further explanations see Table 1)

\begin{tabular}{|c|c|c|c|c|c|}
\hline & $\begin{array}{c}\text { Grados de } \\
\text { libertad }\end{array}$ & $\begin{array}{c}\text { Desvianza } \\
\text { residual }\end{array}$ & $\begin{array}{c}\text { Grados de } \\
\text { libertad residual }\end{array}$ & Desvianza & $\operatorname{Pr}\left(>\chi^{2}\right)$ \\
\hline \multicolumn{6}{|c|}{ Variable respuesta: Presencia/ausencia de larvas competentes de Ch. chorus } \\
\hline Modelo nulo & & & 248 & 311,15 & \\
\hline Año & 1 & 43,34 & 247 & 267,81 & 0,001 \\
\hline Mes & 4 & 74,60 & 243 & 193,21 & 0,001 \\
\hline Localidad & 2 & 13,96 & 241 & 179,26 & 0,001 \\
\hline \multicolumn{6}{|c|}{ Variable respuesta: Densidad positiva de larvas competentes de Ch. chorus } \\
\hline Modelo nulo & & & 78 & 46,48 & \\
\hline Año & 1 & 12,65 & 77 & 33,83 & 0,001 \\
\hline Mes & 4 & 3,85 & 73 & 29,98 & 0,033 \\
\hline Localidad & 2 & 4,62 & 71 & 25,36 & 0,002 \\
\hline
\end{tabular}



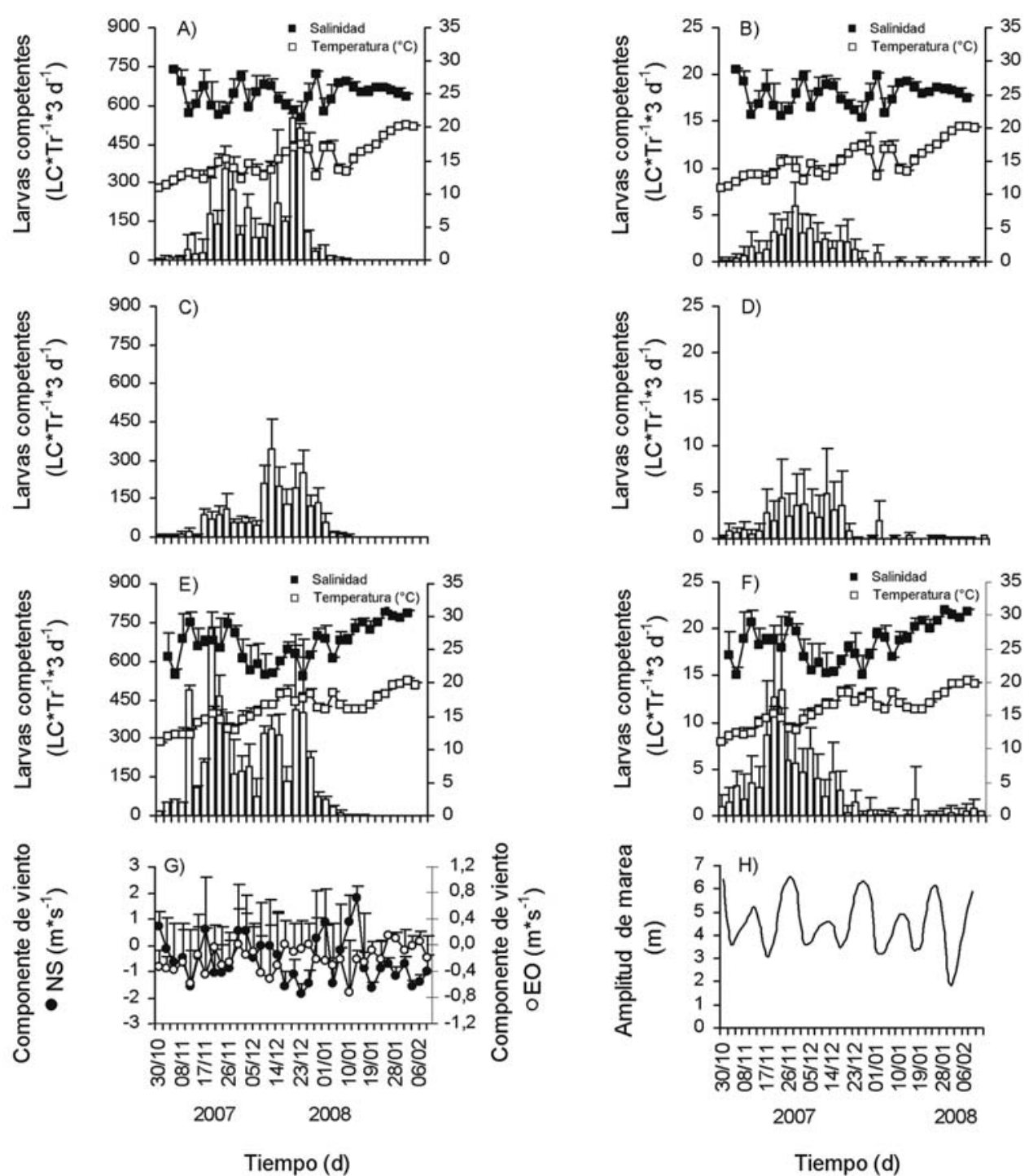

Figura 2. Patrón temporal de salinidad (cuadrado negro), temperatura $\left({ }^{\circ} \mathrm{C}\right)$ (cuadrado blanco) y suministro de larvas competentes (LC) de Mytilus chilensis (columna izquierda) y Choromytilus chorus (columna derecha) (LC Tr $^{-1} 3 \mathrm{~d}^{-1}$ ) (media + DS) en A-B) Pelluhuín, C-D) Quillaipe y E-F) Metri, respectivamente durante la temporada (año) 2007-2008 (T2008). G) Componente de viento NS (círculo negro) y EO (círculo blanco) (media + DS) $\left(\mathrm{m} \mathrm{s}^{-1}\right)$, componente positivo (+): dirección de viento norte o este, componente negativo (-): dirección de viento sur u oeste y H) Amplitud de marea $(\mathrm{m})$ en la costa noreste del Seno de Reloncaví / Seasonal patterns for salinity (black square), temperature $\left({ }^{\circ} \mathrm{C}\right)$ (white square) and supply of competent larvae (LC) of Mytilus chilensis (left column) and Choromytilus chorus (right column) (LC $\left.\operatorname{Tr}^{-1} 3 \mathrm{~d}^{-1}\right)$ (mean + SD) in: A-B) Pelluhuín, C-D) Quillaipe, and E-F) Metri, respectively during the 2007-2008 year (T2008); G) wind components NS (black circle) and EW (white circle) (mean $+\mathrm{SD})\left(\mathrm{m} \mathrm{s}^{-1}\right)$; positive component $(+)$ : wind direction north or east, negative component $(-)$ : wind direction south or west and $\mathrm{H})$ tidal range $(\mathrm{m})$ on the north-east coast of Seno de Reloncaví 

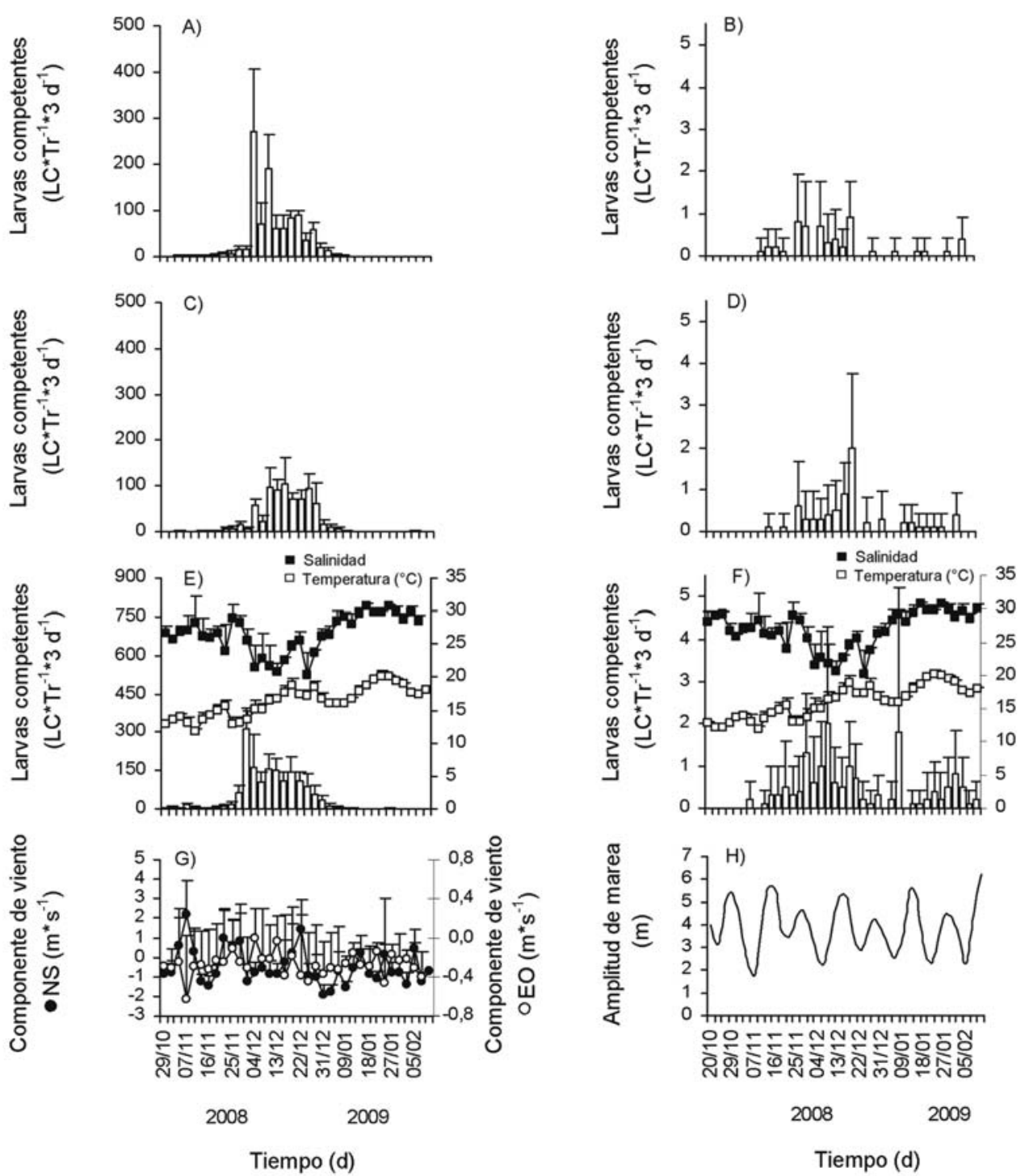

Figura 3. Patrón temporal de salinidad (cuadrado negro), temperatura $\left({ }^{\circ} \mathrm{C}\right)$ (cuadrados blanco) y suministro de larvas competentes (LC) de Mytilus chilensis (columna izquierda) y Choromytilus chorus (columna derecha) (LC $\mathrm{Tr}^{-1} 3 \mathrm{~d}^{-1}$ ) (media + DS) en A-B) Pelluhuín, C-D) Quillaipe y E-F) Metri, respectivamente durante la temporada (año) 2008-2009 (T2009). G) Componente de viento NS (círculo negro) y EO (círculo blanco) (media + DS) $\left(\mathrm{m} \mathrm{s}^{-1}\right)$, componente positivo $(+)$ : dirección de viento norte o este, componente negativo (-): dirección de viento sur u oeste y H) Amplitud de marea $(\mathrm{m})$ en la costa noreste del Seno de Reloncaví / Seasonal patterns for salinity (black square), temperature $\left({ }^{\circ} \mathrm{C}\right)$ (white square) and supply of competent larvae (LC) of Mytilus chilensis (left column) and Choromytilus chorus (right column) (LC $\operatorname{Tr}^{-1} 3 \mathrm{~d}^{-1}$ ) (mean + SD) in: A-B) Pelluhuín, C-D) Quillaipe, and E-F) Metri, respectively during the 2008-2009 year (T2009); G) wind components NS (black circle) and EW (white circle) (mean $+\mathrm{SD})\left(\mathrm{m} \mathrm{s}^{-1}\right)$; positive component (+): wind direction north or east, negative component $(-)$ : wind direction south or west; and $\mathrm{H})$ tidal range $(\mathrm{m})$ on the north-east coast of Seno de Reloncaví 
Durante T2008, predominó el componente de viento sur $(79 \%)$ y oeste $(82 \%)$ con intensidades promedio de $-1,16 \pm 0,48$ y $-0,31 \pm 0,26 \mathrm{~m} \mathrm{~s}^{-1}$, respectivamente (Fig. $2 \mathrm{G}$ ). Durante T2009, el componente de viento sur fue menor tanto en frecuencia (66\%) como intensidad promedio $-0,98 \pm 0,53 \mathrm{~m} \mathrm{~s}^{-1}$ y el promedio de la intensidad del componente de viento oeste fue de $-0,29 \pm 0,11 \mathrm{~m} \mathrm{~s}^{-1}$ (Fig. $3 \mathrm{G})$. La salinidad promedio durante T2008 fue levemente mayor en Metri $(25,9 \pm 2,8)$ que en Pelluhuín $(24,78 \pm 2,1)$, pero no se detectaron diferencias significativas. Para ambas localidades las menores salinidades se registraron entre fines de noviembre y diciembre (Fig. 2 A, E). La temperatura fluctúo entre 17,3 y $11,9^{\circ} \mathrm{C}$ en Pelluhuín y entre 19,4 y $12,2^{\circ} \mathrm{C}$ en Metri (Fig. $2 \mathrm{~A}, \mathrm{E}$ ). La amplitud mareal varió entre $6,4 \mathrm{~m}$ en mareas de sicigia a $1,59 \mathrm{~m}$ en cuadratura (Fig. 2 H) durante T2008. Durante T2009 la salinidad y temperatura promedio registrada en Metri fue de $25,9 \pm 2,9$ y $15,8 \pm 2,1^{\circ} \mathrm{C}$, respectivamente, valores similares a lo observado durante T2008. Menores valores de salinidad (ca., 20) se observaron durante diciembre (Fig. 3 E). El promedio de la amplitud de marea fluctuó entre 5,47 a 1,58 m (Fig. 3 H).

El modelo general lineal mostró que la presencia de las larvas competentes de M. chilensis varió significativamente por efecto del año, mes, localidad y componente este-oeste mostrando mayor probabilidad de presencia de LC en el primer año (T2008), y con predominio de componente este de viento (Tabla 1). La densidad positiva de LC de M. chilensis varió por efecto de las variables año, mes y localidad (Tabla 1).

Por otro lado, la presencia y abundancia de larvas competentes de Ch. chorus varió significativamente por efecto de los variables año, mes y localidad (Tabla 2), mostrando similar patrón al detectado para la presencia y abundancia de LC de M. chilensis, pero con baja frecuencia. La densidad de LC de Ch. chorus fue significativamente distinta solo entre noviembre y febrero (comparación a posteriori de Tukey, $P<0,05$ ), pero la presencia de LC fue distinta en la mayoría de los meses, con excepción de enero, febrero y octubre y este último con noviembre (comparación a posteriori de Tukey, $P>$ 0,05). La presencia de larvas de Ch. chorus fue distinta entre Metri y las otras dos localidades (comparación $a$ posteriori de Tukey, $P<0,05$ ), pero la densidad de larvas fue solo distinta entre las 2 localidades extremas (Metri y Pelluhuín) $(P<0,05)$.

Para el caso específico de M. chilensis en la localidad Metri se observó que el mejor ajuste, se obtuvo cuando se incluyeron las variables año, mes, salinidad y temperatura (Tabla 3). Aunque las variables temperatura y salinidad no fueron significativas para presencia/ ausencia de larvas competentes, ni para densidad positiva para el caso de la salinidad, estas variables fueron consideradas de acuerdo al criterio de información de Akaike, donde su inclusión mejora la predicción del modelo, por lo que las variables se mantuvieron en el modelo final. El componente de viento no fue significativo en este análisis. Larvas competentes fueron más abundantes durante la primera temporada de muestreo (T2008), durante noviembre y asociadas a menores salinidades y temperaturas. El ajuste de este modelo estadístico a la abundancia de LC observada fue mejor durante el segundo año (T2009), explicando el 72\% de la desvianza (Tabla 3, 4).

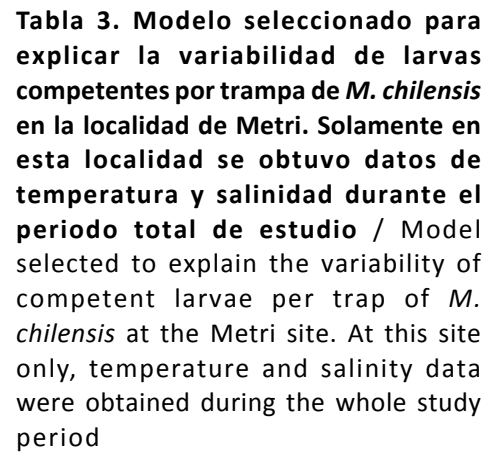

Tabla 3. Modelo seleccionado para explicar la variabilidad de larvas competentes por trampa de $M$. chilensis en la localidad de Metri. Solamente en esta localidad se obtuvo datos de temperatura y salinidad durante el periodo total de estudio / Model selected to explain the variability of competent larvae per trap of $M$. chilensis at the Metri site. At this site only, temperature and salinity data period

\begin{tabular}{ccccc}
\hline $\begin{array}{c}\text { Grados de } \\
\text { libertad }\end{array}$ & $\begin{array}{c}\text { Desvianza } \\
\text { residual }\end{array}$ & $\begin{array}{c}\text { Grados de } \\
\text { libertad residual }\end{array}$ & Desvianza & $\operatorname{Pr}\left(>\chi^{2}\right)$ \\
\hline
\end{tabular}

$\begin{array}{lrrrrr}\text { Variable respuesta: Presencia/ausencia de larvas competentes de } M \text {. chilensis en Metri } \\ \text { Modelo nulo } & 1 & & 82 & 78,433 & \\ \text { Año } & 4 & 34,55 & 81 & 70,881 & 0,006 \\ \text { Mes } & 1 & 3,17 & 77 & 35,961 & 0,001 \\ \text { Temperatura } & 1 & 2,31 & 75 & 32,795 & 0,075 \\ \text { Salinidad } & 1 & & 30,482 & 0,128\end{array}$

Variable respuesta: Densidad positiva de larvas competentes de $M$. chilensis en Metri

\begin{tabular}{|c|c|c|c|c|c|}
\hline Modelo nulo & & & 67 & 211,154 & \\
\hline Año & 1 & 24,08 & 66 & 187,07 & 0,001 \\
\hline Mes & 4 & 118,30 & 62 & 68,769 & 0,001 \\
\hline Temperatura & 1 & 7,33 & 61 & 61,44 & 0,007 \\
\hline Salinidad & 1 & 3,04 & 60 & 58,397 & 0,082 \\
\hline
\end{tabular}


Por otro lado, las larvas competentes de Ch. chorus fueron escasas, y solo las variables año y mes explicaron significativamente su variación en Metri.

Durante T2008 se observó máxima correlación entre la disponibilidad de larvas competentes el mismo día ( $\lambda$ : 0 ; $P<0,05)$ entre todas las localidades estudiadas para ambas especies indicando suministro larval simultáneo en las 3 localidades (Tabla 5). En cambio, durante la T2009, se observó, además del suministro simultáneo, una correlación positiva a los 3 días entre Metri y las otras dos localidades para $M$. chilensis $(\lambda:-1, r: 0,71$ y r: 0,86$)$ y para Ch. chorus entre Metri y Pelluhuín $(\lambda:-1$, r: 0,54), indicando un desfase en la abundancia de LC entre las localidades (Tabla 5).

Tabla 4. Parámetros del modelo ajustado para densidad de larvas competentes de $M$. chilensis en Metri (Larvas competentes= año+mes+temperatura+salinidad). El valor del intercepto es obtenido considerando los factores año: 1 y mes: diciembre. El valor de probabilidad ( $\mathbf{t}$ ) indica la probabilidad que el coeficiente sea igual a $\mathbf{0} /$ Model parameters adjusted for density competent larvae of $M$. chilensis in Metri (Competent larvae = year+month+temperature+salinity). The value of the intercept is obtained considering the factors year: 1 and month: December. The probability value $(t)$ denotes the probability that the coefficient is equal to 0

\begin{tabular}{lcccc}
\hline Coeficiente & Estimado & $\begin{array}{c}\text { Error } \\
\text { estándar }\end{array}$ & Valor $\mathrm{P}$ & Probabilidad $(>|\mathrm{t}|)$ \\
\hline \multicolumn{4}{l}{ Variable respuesta: Densidad positiva } \\
Intercepto & 13,71 & 2,42 & 5,66 & 0,001 \\
Año 2 & $-1,65$ & 0,25 & $-6,64$ & 0,001 \\
Octubre & $-4,35$ & 0,81 & $-5,35$ & 0,001 \\
Noviembre & $-1,17$ & 0,56 & $-2,09$ & 0,041 \\
Enero & $-2,49$ & 0,61 & $-4,07$ & 0,001 \\
Febrero & $-4,01$ & 0,76 & $-5,25$ & 0,001 \\
Temperatura & $-0,25$ & 0,09 & $-2,64$ & 0,010 \\
Salinidad & $-0,15$ & 0,09 & $-1,66$ & 0,102 \\
\hline
\end{tabular}

Tabla 5. Correlaciones cruzadas de la disponibilidad de larvas competentes de Mytilus chilensis y de Choromytilus chorus entre las distintas localidades estudiadas, Pelluhuín, Quillaipe y Metri, en ambos años de muestreo, T2008, T2009. $\lambda$ : lapso, valores negativos indican que la variable 2 se correlaciona con la variable 1 con un retraso en el tiempo, $\lambda$ : -1 indica un retraso de un periodo de muestreo o tres días en la correlación de la variable $\mathbf{2}$ con respecto a la variable 1 . r: coeficiente de correlación. ns: no significativo, $\boldsymbol{P}>\mathbf{0 , 0 5}$ ) / Cross-correlations of competent larvae availability of Mytilus chilensis and Choromytilus chorus between the different sites studied, Pelluhuín, Quillaipe and Metri, in the T2008 and T2009 years. $\lambda$ : lag, negative values indicate that variable 2 correlates with variable 1 with a lag; $\lambda$ : -1 indicates a lag of one sampling period or three days in the correlation of variable 2 with respect to variable 1. r: correlation coefficient. ns: not significant, $P>0.05$ )

\begin{tabular}{|c|c|c|c|c|}
\hline Especie & Localidad & $\lambda$ & $\begin{array}{c}\mathrm{T} 2008 \\
\mathrm{r}\end{array}$ & $\begin{array}{c}\mathrm{T} 2009 \\
\mathrm{r}\end{array}$ \\
\hline \multirow[t]{6}{*}{ M. chilensis } & Metri-Quillaipe & -1 & n.s & 0,71 \\
\hline & & 0 & 0,82 & 0,56 \\
\hline & Metri-Pelluhuín & -1 & n.s & 0,86 \\
\hline & & 0 & 0,64 & 0,56 \\
\hline & Quillaipe-Pelluhuín & -1 & 0,54 & n.s \\
\hline & & 0 & 0,65 & 0,66 \\
\hline \multirow[t]{6}{*}{ Ch. chorus } & Metri-Quillaipe & -1 & 0,75 & n.s \\
\hline & & 0 & 0,83 & n.s \\
\hline & Metri-Pelluhuín & -1 & 0,76 & 0,54 \\
\hline & & 0 & 0,77 & n.s \\
\hline & Quillaipe-Pelluhuín & -1 & 0,77 & n.s \\
\hline & & 0 & 0,87 & 0,65 \\
\hline
\end{tabular}




\section{Discusión}

El suministro larval de Mytilus chilensis y Choromytilus chorus presentó variabilidad temporal y espacial en una extensión de aproximadamente $30 \mathrm{~km}$ en la costa noreste del Seno de Reloncaví. Espacialmente, LC de M. chilensis mostraron mayor densidad en Metri disminuyendo hacia las otras localidades, pero correlacionándose entre sí. Ch. chorus presentó un patrón similar, aunque su abundancia fue significativamente menor.

Aunque la variabilidad en la abundancia de larvas de M. chilensis ha sido estudiada en la zona (Leiva et al. 2007, Marilao 2009, Avendaño et al. 2011), poco se conoce sobre la dinámica de los estadios tempranos de $M$. chilensis y mucho menos de sus procesos de transportes asociados en la zona, elementos considerados clave para entender la disponibilidad de LC en este sistema de mar interior.

La variabilidad espacial y temporal del suministro larval observado en este estudio, asociada principalmente a la variación en el componente de viento este-oeste, a la temperatura y en menor grado a la salinidad sugieren: i) el efecto de variables ambientales afectado la disponibilidad de LC en las zonas de estudio y ii) el potencial transporte de larvas desde el sureste del Seno de Reloncaví hasta al menos la zona de Pelluhuín.

Se han observado variaciones espaciales en el asentamiento en numerosos invertebrados a distintas escalas espaciales $(\mathrm{cm} \mathrm{a} \mathrm{km})$ en una misma región biogeográfica (Petraitis 1991, Bertness et al. 1992, Hunt \& Schiebling 1996). Sitios separados en escalas de kilómetros o menos presentan diferente intensidad en el asentamiento pudiendo relacionarse con diferencias en el suministro larval (Connell 1985, Sutherland 1990, Porri et al. 2006a), como fue observado en el presente estudio, lo que podría reflejar la influencia de características oceanográficas y topográficas locales (Gaines \& Bertness 1992, Connolly et al. 2001, Menge et al. 2003, Porri et al. 2006b, Mc Quaid \& Phillips 2006, Lagos et al. 2007).

Específicamente, en el intermareal de la costa central de Chile se ha observado para Perumytilus purpuratus alta variabilidad espacial asociada a procesos de micro y mesoescala (Lagos et al. 2007). Adicionalmente, se ha documentado que el asentamiento en la familia Mytilidae es afectado por las condiciones de flujo de microescala (Cáceres-Martínez et al. 1994, Pernet et al. 2003) y por la amplitud mareal (Hunt \& Scheibling 1996).

En este estudio la presencia de LC de M. chilensis y Ch. chorus fue afectada por la variable mes, año y localidad, y adicionalmente para $M$. chilensis la variable componente de viento este-oeste, registrada de manera global para toda el área de estudio, sugiriendo variaciones anuales, mensuales y locales en la disponibilidad de LC de ambas especies. Sin embargo, la magnitud de la disponibilidad de larvas competentes de M. chilensis, no fue afectada por el componente de viento sugiriendo variaciones locales en los procesos que modulan el suministro larval de las especies estudiadas. En el caso de Ch. chorus la magnitud de larvas competentes disponibles también fue afectada por la variación anual, mensual y local.

La presencia de LC de $M$. chilensis en la localidad Metri estuvo asociada sólo a la variación temporal (anual y mensual), mientras que la variación en la abundancia de larvas competentes estuvo asociada a la temperatura y salinidad. Aunque esta última variable no explica por sí sola una fracción significativa de la desvianza del modelo, en presencia de la variable temperatura, y considerando el AIC y su contribución en mejorar la predicción del modelo estadístico, se optó por mantenerla en el modelo. Así una menor temperatura y salinidad explican una mayor disponibilidad de LC en Metri, lo que sugiere la intrusión de agua mas estuarina que podría estar transportando larvas hacia esta localidad probablemente desde el estuario Reloncaví cuya desembocadura se encuentra a $13 \mathrm{~km}$ aproximadamente al sureste de Metri. En este estuario se han descrito extensos bancos de $M$. chilensis (Lizama 2003르), además de la presencia de centros de cultivo de esta especie los que podrían estar actuando como fuente de larvas para el área estudiada, considerando los rasgos de la circulación descritos por Gebauer (2004). Lo anterior, es consistente con lo sugerido por Valle-Levinson et al. (2007) quienes proponen que en términos de una partícula boyante (e.g., larva planctónica) viajando los $55 \mathrm{~km}$ de fiordo a $5 \mathrm{~cm} \mathrm{~s}^{-1}$, la partícula tomaría en promedio 12 a 13 días desde la cabecera hasta la boca del fiordo. Considerando que el desarrollo larval de $M$. chilensis dura 19 a 45 días (Toro \& Sastre 1995, Toro et al. 2004, Ruiz et al. 2008) es altamente probable que el suministro de larvas de $M$. chilensis en Metri dependa en alguna magnitud del aporte de larvas desde el estuario.

${ }^{1}$ Lizama O. 2003. Catastro de bancos naturales en el estuario Reloncaví. Servicio País, pp. 42, Cochamo. 
Ya que la disponibilidad de larvas competentes de $M$. chilensis en Metri estuvo positivamente correlacionada con la disponibilidad de LC en Quillaipe y Pelluhuín, es probable que este sistema sea dependiente de la producción de larvas del estuario Reloncaví y de eventos de transporte larval común para todas las localidades, por ejemplo los generados por el componente de viento este. Un patrón similar ha sido propuesto para explicar variaciones en la abundancia de larvas megalopas, de Carcinus maenas en la costa oeste de Portugal, atribuyéndose estas variaciones a la distribución de la abundancia de las poblaciones y al flujo general de viento sur durante la temporada de presencia de larvas en la costa (Queiroga et al. 2006).

Diferentes mecanismos de transporte han sido propuestos para explicar la sincronía en el asentamiento en invertebrados marinos, por ejemplo: i) ondas internas en escalas espaciales entre 30 y $300 \mathrm{~m}$ en cirripedios en el norte de Baja California (Ladah et al. 2005), ii) surgencia a mayores distancias $(<30 \mathrm{~km})$ para distintas especies de cirripedios y para el bivalvo Perumytilus purpuratus en la costa central de Chile (Lagos et al. 2007) y, iii) aspiraciones de Bernoulli en larvas de Concholepas concholepas (Molinet et al. 2006). Además, la correlación espacial en el asentamiento en escalas desde 100s de m a $\mathrm{km}$ ha sido asociada a mecanismos de retención y advección, asociados a vientos permanentes hacia la costa a través de extensas áreas costeras (Bertness et al. 1996, Jeffrey \& Underwood 2000).

Aunque el modelo estadístico predice moderadamente bien la presencia y magnitud del suministro larval de $M$. chilensis en Metri, durante la primera temporada, la predicción está desfasada en aproximadamente 24 días. Esto implica que existen otras variables probablemente asociadas a procesos locales ya sea de Metri (producción larval local), como a procesos locales en el estuario Reloncaví u otro sector aledaño, lo que también estaría afectando el transporte de larvas hacia la zona estudiada. Finalmente, considerando que larvas de $M$. chilensis como de otros mitílidos se ubican principalmente en la capa superficial de la columna de agua (Buzeta et al. 1998, Garland et al. 2002, McQuaid \& Phillips 2006), es altamente probable que su advección sea afectada por vientos, lo que se evidenció en el presente trabajo, y por variaciones en la descarga de ríos y precipitaciones, lo que debe ser estudiado en mayor profundidad.

\section{Agradecimientos}

Esta investigación fue financiada por el proyecto DIUniversidad de Los Lagos (0707) y Programa Bicentenario de Ciencia y Tecnología (Ecología y Nutrición larval).

\section{LITERATURA CITADA}

Aitchison J. 1955. On the distribution of a positive random variable having a discrete probability mass at the origin. Journal of the American Statistical Association 50: 901908.

Ardisson PL \& E Bourget. 1997. A study of the relationship between freshwater runoff and benthos abundance: a scaleoriented approach. Estuarine, Coastal and Shelf Science 45: 535-545.

Avendaño M, M Cantillánez, M Le Pennec, C Varela \& C Garcias. 2011. Distribución temporal de larvas de Mytilus chilensis (Hupé, 1954) (Mollusca: Mytilidae), en el mar interior de Chiloé, sur de Chile. Latin American Journal of Aquatic Research 39(3): 416-426.

Bellolio G, P Toledo \& E Dupré. 1996. Desarrollo larvario de Choromytilus chorus en condiciones de laboratorio. Scientia Marina 60(2-3): 353-360.

Bertness MD, SD Gaines, EG Stephens \& PO Yund. 1992. Components of recruitment in populations of the acorn barnacle Semibalanus balanoides (Linnaeus). Journal of Experimental Marine Biology and Ecology 156: 199-215.

Bertness MD, SD Gaines \& RA Wahle. 1996. Wind-driven settlement patterns in the acorn barnacle Semibalanus balanoides. Marine Ecology Progress Series 137: 103-110.

Burnham KP \& DR Anderson. 2004. Multimodel inference: Understanding AIC and BIC in model selection. Sociological Methods \& Research 33(2): 261-304.

Buzeta, R, JE Winter, OR Chaparro, J Toro, M Sanhueza, M Matamala, A Gutiérrez, JM Navarro \& H Arriagada. 1988. Desarrollo de la maricultura en Chiloé, Chile, 147 pp. Universidad Austral de Chile, Centro Internacional de Investigaciones para el Desarrollo (CIID-Canada), Valdivia.

Cáceres-Martínez J, JAF Robledo \& A Figueras. 1994. Settlement and post-larvae behaviour of Mytilus galloprovincialis: field and laboratory experiments. Marine Ecology Progress Series 112: 107-117.

Cameron RA \& SC Schroter. 1980. Sea urchin recruitment: Effect of substrate on juvenile distribution. Marine Ecology Progress Series 2: 243-247.

Chaparro OR \& JE Winter. 1983. The effect of winter period, gametogenesis and spawning on the calorific content of soft parts in Mytilus chilensis. Aquaculture 32: 419-422.

Chatfield C. 2004. The analysis of time series, 333 pp. Chapman \& Hall, London.

Connell JH. 1985. The consequences of variation in initial settlement vs. post-settlement mortality in rocky intertidal communities. Journal of Experimental Marine Biology and Ecology 93: 11-45. 
Connolly SR, BA Menge \& J Roughgarden. 2001. A latitudinal gradient in recruitment of intertidal invertebrates in the northeast Pacific Ocean. Ecology 82: 1799-1813.

Dávila PM, D Figueroa \& E Müller. 2002. Freshwater input into the coastal ocean and its relation with the salinity

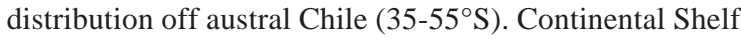
Research 22: 521-534.

Dobretsov S \& G Miron. 2001. Larval and postlarval vertical distribution of the mussel Mytilus edulis in the White Sea. Marine Ecology Progress Series 218: 179-187.

Emery WJ \& RE Thomson. 1997. Data analysis methods in physical oceanography, 634 pp. Pergamon, Great Britain.

Fox J \& W Sanford. 2010. Car: Companion to Applied Regression. R package version 2.0-2. [on line] <http:// CRAN.R-project.org/package $=$ car $>$

Gaines SD \& MD Bertness. 1992. Dispersal of juveniles and variable recruitment in sessile marine species. Nature 360: 579-580.

Garland ED, CA Zimmer \& SJ Lentz. 2002. Larval distributions in inner-shelf waters: the roles of wind-driven cross-shelf currents and diel vertical migrations. Limnology and Oceanography 47(3): 803-817.

Gebauer P. 2004. Factores físicos y biológicos involucrados en el cierre del eslabón larval y reclutamiento de Petrolisthes laevigatus (Guerin, 1835) (Decapoda: Porcellanidae) en el Seno de Reloncaví. Tesis Doctoral, Facultad de Ciencias, Universidad Austral de Chile, Valdivia, 99 pp.

Harrold C, S Lisin, KH Light \& S Tudor. 1991. Isolating settlement from recruitment of sea urchins. Journal of Experimental Marine Biology and Ecology 147: 81-94.

Hothorn T, F Bretz \& P Westfall. 2008. Simultaneous inference in general parametric models. Biometrical Journal 50(3): 346-363.

Hunt HL \& RE Scheibling. 1996. Physical and biological factors influencing mussel (Mytilus trossulus, M. edulis) settlement on a wave-exposed rocky shore. Marine Ecology Progress Series 142: 1-3.

Iriarte JL, HE González, KK Liu, C Rivas \& C Valenzuela. 2007. Spatial and temporal variability of chlorophyll and primary productivity in surface waters of southern Chile (41.5$43^{\circ}$ S). Estuarine, Coastal and Shelf Science 74: 471-480.

Jeffrey CJ \& AJ Underwood. 2000. Consistent spatial patterns of arrival of larvae of the honeycomb barnacle Chaemaesipho tasmanica Foster and Anderson in New South Wales. Journal Experimental Marine Biology and Ecology 252: 109-117.

Kleinbaum DG, LL Kupper, KE Muller \& A Nizam. 1998. Applied regression analysis and other multivariate methods, 906 pp. Publishing Company, Pacific Grove.

Ladah LB, FJ Tapia, J Pineda \& M López. 2005. Spatial heterogeneous, synchronous settlement of Chthamalus spp. larvae in northern Baja California. Marine Ecology Progress Series 302: 177-185.
Lagos NA, FJ Tapia, SA Navarrete \& JC Castilla. 2007. Spatial synchrony in the recruitment of intertidal invertebrates along the coast of central Chile. Marine Ecology Progress Series 350: 29-39.

Legendre P \& L Legendre. 1998. Numerical ecology, 853 pp. Elsevier Science B.V, Amsterdam.

Leiva G, C Santibañez, JL Bartheld, C Molinet \& JM Navarro. 2007. Definición de criterios biológicos, ambientales, sanitarios y operativos para la instalación de colectores de moluscos bivalvos en la X región. Informe Final FIP 2005-18: 1-197. [en línea] <http://www.fip.cl/ FIP/Archivos/pdf/informes/inffinal\%202005-18.pdf>

Lorenzen S, C Gallardo, C Jara, E Classing, G Pequeño \& C Moreno. 1979. Mariscos y peces de importancia comercial en el sur de Chile, pp. 24-25. Universidad Austral de Chile, Valdivia.

Mann R, BM Campos \& MW Luckenbach. 1991. Swimming rate and responses of larvae of three mactrid bivalves to salinity discontinuities. Marine Ecology Progress Series 68: 257-269.

Marilao G. 2009. Distribución espacial y temporal de estadíos larvales tempranos de mitílidos en Bahía Ilque (4138'20”'s; $73^{\circ} 05^{\prime} 00^{\prime}$ W), X región, Chile. Tesis de Ingeniería en Acuicultura, Instituto de Acuicultura, Universidad Austral de Chile, Puerto Montt, 62 pp.

McCullagh P \& JA Nedler. 1989. General linear models, 532 pp. Chapman and Hall, London.

McQuaid CD \& TE Phillips. 2006. Mesoscale variation in reproduction, recruitment and population structure of intertidal mussels with low larval input: a bay/open coast comparison. Marine Ecology Progress Series 327: 193-206.

Menge BA, J Lubchenco, MES Bracken, F Chan, MM Foley, TL Freidenburg, SD Gaines, G Hudson, C Krenz, H Leslie, DNL Menge, R Russel \& MS Websyer. 2003. Coastal oceanography sets the pace of rocky intertidal community dynamics. Proceedings of the National Academic of Sciences 100(21): 12229-12234 of United States.

Molinet C, A Valle-Levinson, CA Moreno, M Cáceres, M Bello \& M Castillo. 2006. Effects of sill processes on the distribution of epineustonic competent larvae in a stratified system of Southern Chile. Marine Ecology Progress Series 324: 95-104.

Orensanz JM, A Parma, T Turk \& J Valero. 2006. Dynamics, assessments and management of exploited natural population. En: Shunway S \& GJ Parsons (eds). Scallops: Biology, ecology and aquaculture, pp. 765-868. Elsevier. Amsterdam.

Pennington JT. 1983. Efficient estimators of abundance for fish and plankton surveys. Biometrics 39: 281-286.

Pernet F, R Tremblay \& E Bourget. 2003. Settlement success, spatial pattern and behavior of mussel larvae Mytilus spp. in experimental ‘downwelling' systems of varying velocity and turbulence. Marine Ecology Progress Series 260: 125-140. 
Petraitis PS. 1991. Recruitment of the mussel Mytilus edulis L. in sheltered and exposed shores in Maine, U. S. A. Journal of Experimental Marine Biology and Ecology 147: 65-80.

Pickard GL. 1971. Some physical oceanographic features of inlets of Chile. Journal of the Fisheries Research Board of Canada 28: 1077-1106.

Pineda J. 2000. Linking larval settlement to larval transport: assumptions, potential and pitfalls. Oceanography of the Eastern Pacific 1: 84-105.

Pineda J, F Porri, V Starcazak \& J Blythe. 2010. Causes of decoupling between larval supply and settlement and consequences for understanding recruitment and connectivity. Journal of Experimental Marine Biology and Ecology 392: 9-21.

Porri F, CD McQuaid \& S Radloff. 2006a. Spatio-temporal variability of larval abundance and settlement of Perna perna: differential delivery of mussels. Marine Ecology Progress Series 315: 141-150.

Porri F, CD McQuaid \& S Radloff. 2006b. Temporal scales of variation in settlement and recruitment of the mussel Perna perna (Linnaeus, 1758). Journal of Experimental Marine Biology and Ecology 332: 178-187.

Queiroga H, MJ Almeida, T Alpium, AAV Flores, S Francisco, I González-Gordillo, AI Miranda, I Silva \& J Paula. 2006. Tide and wind control of megalopal supply to estuarine crab populations on the Portuguese west coast. Marine Ecology Progress Series 307: 21-36.

R Development Core Team. 2011. R: A language and environment for statistical computing. R Foundation for Statistical Computing, Vienna, Austria. [on line] <http:// www.R-project.org>

Ramorino L \& B Campos. 1993. Larvas y postlarvas de Mytilidae (Mollusca: Bivalvia). Revista de Biología Marina 19(2): 143-192.

Rilov G, SE Dudas, BA Menge, BA Grantham, J Lubchenco \& DR Schiel. 2008. The surf zone: a semi-permeable barrier to onshore recruitment of invertebrate larvae? Journal of Experimental Marine Biology and Ecology 361: 59-74.

Ruiz M, E Tarifeño, A Llanos-Rivera, C Padget \& B Campos. 2008. Efecto de la temperatura en el desarrollo embrionario y larval del mejillón, Mytilus galloprovincialis (Lamarck, 1819). Revista de Biología Marina y Oceanografía 43(1): 51-61.

SERNAPESCA. 2010. Anuario estadístico de pesca. Servicio Nacional de Pesca y Acuicultura, Ministerio de Economía, Fomento y Turismo, Valparaíso. [en línea] <www.sernapesca.cl>
Shanks AL. 1995. Mechanisms of cross-shelf dispersal of larval invertebrates and fish. In: McEdward L (ed). Ecology of marine invertebrate larvae, pp. 323-367. CRC Press, Boca Raton.

Silva N, HA Sievers \& R Prado. 1995. Características oceanográficas y una proposición de circulación, para algunos canales australes de Chile entre $41^{\circ} 20^{\prime} \mathrm{S}, 46^{\circ} 40^{\prime}$ 'S. Revista de Biología Marina 30(2): 207-254.

Silva N, C Calvete \& HA Sievers. 1997. Características oceanográficas físicas y químicas de canales australes chilenos entre Puerto Montt y laguna San Rafael (Crucero CIMAR-Fiordo 1). Ciencia y Tecnología del Mar 20: 23106.

Silva N, C Calvete \& HA Sievers. 1998. Masas de agua y circulación general para algunos canales australes entre Puerto Montt y laguna San Rafael, Chile (Crucero CimarFiordo 1). Ciencia y Tecnología del Mar 21: 17-48.

Stefánsson G. 1996. Analysis of groundfish survey abundance data: combining the GLM and delta aprroaches. ICES Journal of Marine Science 53: 577-588.

Sutherland JP. 1990. Recruitment regulates demographic variation in a tropical intertidal barnacle. Ecology 71: 955972.

Todd CD. 1998. Larval supply and recruitment of benthic invertebrates: do larvae disperse as far as we believe? Hidrobiologia 375/376: 1-21.

Toro JE \& D Sastre. 1995. Induced triploidy in the Chilean blue mussel, Mytilus chilensis (Hupe 1854), and performance of triploid larvae. Journal of Shellfish Research 14: 161-164.

Toro JE, AC Alcapán, AM Vergara \& JA Ojeda. 2004. Heritability estimates of larval and spat shell height in the Chilean blue mussel (Mytilus chilensis Hupe 1854) produced under controlled laboratory conditions. Aquatic Research 35: 56-61.

Valle-Levinson A, N Sarkar, R Sana, D Soto \& J León. 2007. Spatial structure of hydrography and flow in a Chilean fjord, Estuario Reloncaví. Estuaries and Coasts 30: 113-126.

Wolfinger R \& M O’Connell. 1993. Generalized linear mixed models: a pseudo likelihood approach. Journal of Statistical Computation and Simulation 4: 233-243.

Young CM. 1995. Behavior and locomotion during the dispersal phase of larval life. In: McEdward L (ed). Ecology of marine invertebrate larvae, pp. 249-277. CRC Press, Boca Raton.

Zagal C \& C Hermosilla. 2001. Guía de invertebrados marinos del litoral valdiviano, 217 pp. Quebecor Wild Chile, Santiago de Chile. 